\title{
Parallel Soft Spherical Detection for Coded MIMO Systems
}

\author{
Hosein Nikopour ${ }^{\dagger}$, Amir K. Khandani ${ }^{\dagger}$, and Aladdin Saleh ${ }^{\ddagger}$ \\ ${ }^{\dagger}$ Department of Electrical and Computer Engineering, University of Waterloo \\ 200 University Ave. W., Waterloo, ON N2L 3G1, Canada \\ Telephone: (519) 885-1211 x5324, Fax: (519) 888-4338 \\ Email: \{hossein, khandani\}@cst.uwaterloo.ca \\ $\ddagger$ Wireless Technology, Bell Canada \\ Email: aladdin.saleh@bell.ca
}

\begin{abstract}
A sub-optimum a-posteriori probability (APP) detector is proposed for iterative joint detection/decoding in a multiple-input multiple-output (MIMO) wireless communication system employing an outer code. The proposed detector searches inside a given sphere in a parallel manner to simultaneously find a list of $m$-best points based on an additive metric. The metric is formed by combining the channel output and the a-priori information. The parallel structure of the proposed method is suitable for hardware parallelization. The radius of the sphere and the value of $m$ are selected according to the channel condition to reduce the complexity. Numerical results are provided showing a significant reduction in the average complexity (for a similar performance and peak complexity) as compared to the best earlier known method. The proposed scheme is applied for the decoding of the rate $2,4 \times 2$ MIMO code employed in the $802.16 \mathrm{e}$ standard.
\end{abstract}

\section{INTRODUCTION}

Recently, there has been considerable interest in multiple transmit and receive antennas because of capability to offer a high data rate over fading channels [1], [2]. The Bell-Lab layered space-time (BLAST) [3] is an example of MIMO systems. In MIMO channels, as the received signal set has a regular structure, lattice decoding methods can be used for detection. However, the complexity of the optimum lattice decoding grows exponentially with the number of transmit antennas, and with the constellation size. Several sub-optimum MIMO detectors have been proposed based on nulling and interference cancellation (IC) [3], [4], which essentially perform zero-forcing or minimum-mean-square-error equalization. The performance of such detectors is significantly inferior to that of the ML detection.

Sphere decoder (SD) [5], [6] is used as a detection method for MIMO systems with near ML performance. In SD, the lattice points inside a hyper-sphere are checked and the closest lattice point is determined. It is known that even the average complexity of the SD algorithm is exponential [7].

The capacity of MIMO systems can not be achieved unless by using an outer channel code concatenated with the spacetime mapper acting like an inner code. Iterative a posteriori probability (APP) detection techniques [8], [9], such as iterative joint detection and decoding with soft inputs and outputs [10]-[13], can be used for decoding of such systems.
In contrast to the ML detector which finds the closest valid point to the received noisy signal, the iterative soft MIMO detector provides probabilistic information about the transmitted bits. This soft information is passed to the decoder for the underlying error correction code (ECC), such as turbo [14] or low-density parity check (LDPC) [15] code. The soft outputs of the decoder may be used as new soft inputs for the MIMO detector, and hence this method can work in an iterative fashion to improve the performance.

The optimum APP MIMO detector which enumerates all the signal points for the soft metric computation has a very large complexity [10]. To reduce the complexity, several schemes are proposed based on finding a small set (list) of highly probable points for computing the soft values. List sphere decoder (LSD) [16] is a method in this category which uses a list of candidates inside a preset sphere for computing the soft information. The main drawback in the known LSD methods is the instability of the list size and the associated problem of the radius selection and reduction during the search. This significantly increases the complexity as compared to the original hard SD (HSD). In [17], this problem is addressed by building a spherical list centered around the ML point, instead of the received point. Although the effective list size is well controlled, the size should still be set at a large value to achieve a reasonable performance.

Stack algorithm with limited stack size is another list detector proposed under different titles such as list-sequential (LISS) detector [18], deterministic sequential Monte Carlo (SMC) [19], or sequential tree search [20], [21]. This scheme evaluates only the 'good' candidate vectors with the aid of a sequential tree searching scheme, which is based on the $m$-algorithm [22], [23]. The disadvantage of this class of detectors is that the complexity is only a function of the stack size and is independent of the received SNR.

Soft-to-hard transformation is another approach [24] to build a soft detector. The transformation converts a soft detection problem to a set of hard detection problems, which are less complex compared with the LSD. This approach, however, imposes some limitations on the underlying modulation and coding schemes. Therefore, this approach can not be considered as a general solution for the soft MIMO detection. 

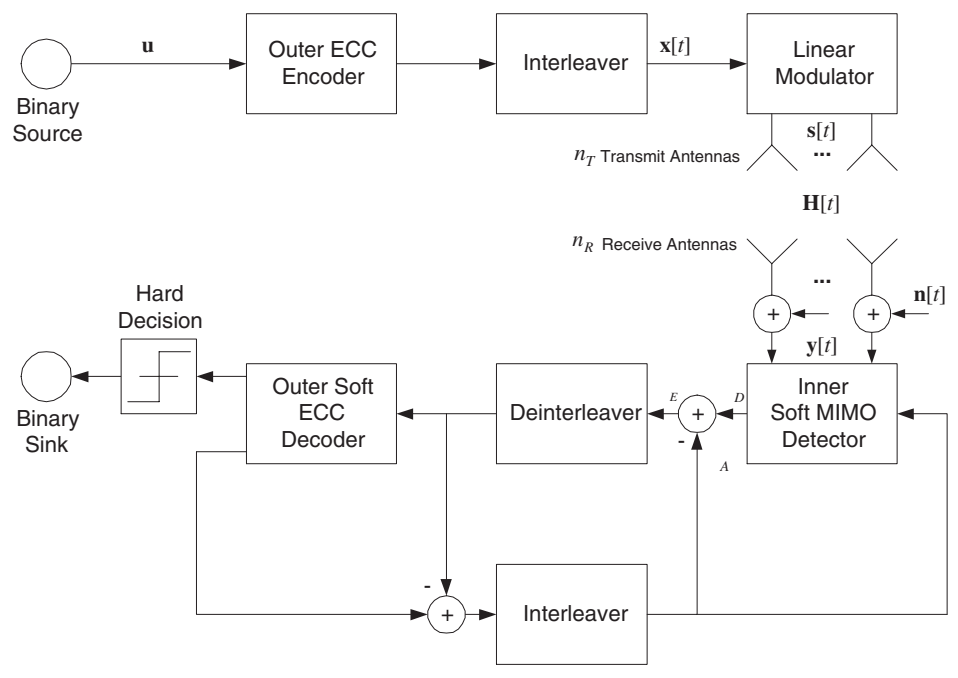

Fig. 1. MIMO transmitter and iterative receiver.

An alternative algorithm is also developed with polynomial average complexity which is based on the semi-definite programming (SDP) [25], [26]. The exponential worst case complexity is still unsolvable by this approach.

The main contribution of this work is a low-complexity list MIMO detector based on combining sphere and m-algorithm approaches. First, the traditional SD is modified to work in a parallel manner. The parallel sphere decoder (PSD) grows all the nodes at a given level of the tree simultaneously to find the best paths. During the parallel search, the m-algorithm helps the PSD to reduce the complexity by eliminating some branches. The radius of the sphere and the value of $m$ are selected according to the channel condition to reduce the complexity. The proposed PSD has the benefits of the both sphere and m-algorithm detectors, while it avoids their drawbacks. Numerical results are provided showing a significant reduction in the average complexity (for a similar performance and peak complexity) as compared to the best earlier known methods reported in [16] and [21].

After introducing the MIMO system model under consideration and the iterative receiver in Section II, the proposed sub-optimal MIMO detector is described in Section III. The details of the proposed parallel list detector is presented in Section IV. Section V is devoted to some numerical results, followed by some concluding remarks in Section VI.

\section{MIMO Model AND ITERATIVE Receiver}

We consider a typical bit interleaved coded modulation (BICM) MIMO system [27] with iterative APP receiver as shown in Fig. 1.

A block of information bits $\mathbf{u}$ is encoded by a rate $R$ outer code $\mathcal{C}$. Then, the encoded stream is permuted by an interleaver. At time $t$, the bits of the sequence $\mathbf{x}[t]=$ $\left(x_{1}[t], \ldots, x_{M_{c} n_{T}}[t]\right)^{\top}$ as a part of the permuted stream are mapped onto a complex vector $\mathbf{s}[t]=\left(s_{1}[t], \ldots, s_{n_{T}}[t]\right)^{\top}$ by $n_{T}$ linear modulators. Each element $s_{i}[t]$ is taken from a complex constellation $\mathcal{Q}$ composed of $2^{M_{c}}$ distinct points. The output of the modulators are passed into a narrow-band multiple antenna channel with $n_{T}$ transmit antennas and $n_{R}$ receive antennas. Throughout this article, it is assumed that $n_{T}=n_{R}$.

Let $\mathbf{y}[t]=\left(y_{1}[t], \ldots, y_{n_{R}}[t]\right)^{\top}$ be an $n_{R}$-dimensional vector of received signal given by,

$$
\mathbf{y}[t]=\mathbf{H}[t] \mathbf{s}[t]+\mathbf{n}[t]
$$

where $\mathbf{H}[t]$ is a matrix of dimension $n_{R} \times n_{T}$ with entries that are independent samples of a complex Gaussian random variable with zeros mean and unit variance (Rayleigh fading). We assume that $\mathbf{H}[t]$ is known to the receiver. Entries of $\mathbf{n}[t]$ are the spatially and temporally complex white Gaussian noise with variance $\sigma^{2}$ per real component. Assuming $\mathrm{E}\left\|s_{i}[t]\right\|^{2}=$ $\rho / n_{T}$ makes the total transmit power equal to $\rho$ [16]. The overall bandwidth efficiency of such a system is $R M_{c} n_{T}$ bits per channel use.

Since the bits among the blocks $\mathbf{x}[t], t=1,2, \ldots$ are outputs of an ECC encoder that introduces redundancy, the block-by-block decision on the bits is no longer optimal. The APP MIMO detector should make decision jointly on all blocks using the knowledge of the correlation across blocks, and the channel code should decode using soft information on all the blocks obtained from the APP MIMO detector. Therefore, an iterative receiver that performs joint detection and decoding is needed.

An iterative receiver consists of two stages: the soft MIMO APP detector, followed by a soft ECC decoder. The two stages are separated by a deinterleaver and an interleaver. Fig. 1 illustrates how the soft information is iterated between the MIMO APP detector and the soft ECC decoder.

As the MIMO APP detector operates on a per-symbol vector basis, we omit the time index and work on the vector $\mathrm{x}$. The optimal log-likelihood ratio (LLR) of the bit $x_{k}, k=$ $1, \ldots, M_{c} n_{T}$, knowing the received vector $\mathbf{y}$, is obtained by 
the APP detector as follows [9]

$$
\lambda_{D}\left(x_{k} \mid \mathbf{y}\right)=\ln \frac{P\left(x_{k}=+1 \mid \mathbf{y}\right)}{P\left(x_{k}=-1 \mid \mathbf{y}\right)}
$$

which constitutes the posterior information about $x_{k}$. Due to the existence of the interleaver, the bits within $\mathrm{x}$ are approximately statistically independent. Employing Bayes' theorem, exploiting the independence among the entries of $\mathbf{x}$, and considering the channel model (1), the soft output values are given by

$$
\lambda_{D}\left(x_{k} \mid \mathbf{y}\right)=\ln \frac{\sum_{\mathbf{x} \in \mathbb{X}_{k}^{+}} \exp \left(\Lambda\left(\mathbf{x}, \boldsymbol{\lambda}_{A} ; \mathbf{y}, \mathbf{H}\right)\right)}{\sum_{\mathbf{x} \in \mathbb{X}_{k}^{-}} \exp \left(\Lambda\left(\mathbf{x}, \boldsymbol{\lambda}_{A} ; \mathbf{y}, \mathbf{H}\right)\right)}
$$

where

$$
\Lambda\left(\mathbf{x}, \boldsymbol{\lambda}_{A} ; \mathbf{y}, \mathbf{H}\right)=-\frac{1}{2 \sigma^{2}}\|\mathbf{y}-\mathbf{H} \mathbf{s}\|^{2}+\frac{1}{2} \mathbf{x}^{\top} \boldsymbol{\lambda}_{A}
$$

and $\mathbb{X}_{k}^{ \pm}=\left\{\mathbf{x} \mid x_{k}= \pm 1\right\}$. With $\lambda_{A}\left(x_{k}\right)=\ln \frac{P\left(x_{k}=+1\right)}{P\left(x_{k}=-1\right)}$ denoting the a priori information of $x_{k}$, we define $\lambda_{A}=$ $\left(\lambda_{A}\left(x_{1}\right), \ldots, \lambda_{A}\left(x_{M_{c} n_{T}}\right)\right)^{\top}$. The a priori information is received from the previous iteration as the soft output of the ECC encoder. By definition, the extrinsic information of $x_{k}$ is given by $\lambda_{E}\left(x_{k} \mid \mathbf{y}\right)=\lambda_{D}\left(x_{k} \mid \mathbf{y}\right)-\lambda_{A}\left(x_{k}\right)$.

The ECC decoder uses the extrinsic information provided by the MIMO detector to update its knowledge about the transmitted bits. The new soft information of the bits serves as the prior information for the next iteration.

\section{Parallel List Sphere Decoder}

The number of vectors $\mathbf{x}$ in the set $\mathbb{X}$ is equal to $2^{M_{c} n_{T}}$. Therefore, noting (3), the complexity of computing $\lambda_{D}\left(x_{k} \mid \mathbf{y}\right)$ is exponential in the length of the bit vector $\mathbf{x}$.

The empirical observation shows that the vast majority of the elements of $\mathbb{X}$ contribute a negligible amount to the summations in (3). We decrease the complexity of the calculation of $\lambda_{D}\left(x_{k} \mid \mathbf{y}\right)$ by enumerating only the high- $\Lambda$-value subset denoted as $\mathcal{L} \subset \mathbb{X}$. The list detection problem is formulated as follows. Find the set of points $\mathcal{L} \subset \mathbb{X}$, with cardinality $|\mathcal{L}|=m<2^{M_{c} n_{T}}$ with the highest $\Lambda$-values [28],

$$
\mathcal{L}=\arg \max _{\substack{\mathcal{L}^{\prime} \subset \mathbb{X} \\\left|\mathcal{L}^{\prime}\right|=m}} \min _{\mathbf{x} \in \mathcal{L}^{\prime}} \Lambda\left(\mathbf{x}, \boldsymbol{\lambda}_{A} ; \mathbf{y}, \mathbf{H}\right) .
$$

The problem of finding the optimum list is in general NPhard [29]. In the following, we will show how the parallel SD approach can be used to find a 'good' list inside a sphere of a given radius.

According to (4), in the absence of a priori information, the required candidate points of the list may be defined as follows,

$$
\forall \mathbf{x}^{\prime} \notin \mathcal{L}, \forall \mathbf{x} \in \mathcal{L}, \quad\|\mathbf{y}-\mathbf{H} \mathbf{s}\|^{2} \ll\left\|\mathbf{y}-\mathbf{H s}^{\prime}\right\|^{2} .
$$

Thus, $\mathcal{L}$ contains points inside a sphere centered at the received point $\mathbf{y}$. Although this list contains the closet points to the received vector $\mathbf{y}$, it does not guarantee to contain the points with the highest $\Lambda$-values. This means the search algorithm should consider both the distance and the a priori information to find the high $\Lambda$-value points.

A traditional HSD searches the closet point in a serial manner [6] based on the distance metric. Starting with an initial radius, the SD finds a candidate point and updates the sphere radius which can not exceed the initial radius. After that, the SD starts the search process over, using the newly computed radius to find any better candidate points. This process continues until finding the closet point. In the LSD [16], one should find a list of points within a preset sphere, and hence, the radius cannot be decreased during the search process. Note that when the radius is fixed, there will be no difference between the complexity of the serial and the parallel search schemes. The proposed parallel scheme, which is called PSD, grows all the branches inside the sphere simultaneously to find all the candidate points. The main advantage of the parallel scheme is that at each level of the sequential tree growing, like the m-algorithm approach (e.g. [18]), we can limit our search to the signal paths with the highest $\Lambda$-values. The additive form of the $\Lambda$-value allows us to calculate the branch metrics sequentially in a tree structure. At each level of the tree growing, existing nodes are explored inside the sphere, the new extended branches are ordered, and finally, the $m$-best branches are selected and the rest are eliminated. Unlike [20] and [21] that use a fixed $m$, here, we select $m$ according to the channel matrix as will be explained later.

\section{Details of the Parallel List Detection ALGORITHM}

The details of the proposed PSD are given in the following.

\section{A. Extension of the Complex Space to the Real Space}

In order to use the SD, first we need to extend the complex model of (1) to the real space [6]. The channel matrix is changed to the real matrix $\mathbf{H} \leftarrow\left(\mathbf{H}_{i j}\right)$ using,

$$
\mathbf{H}_{i j}=\left(\begin{array}{cc}
\Re h_{i j} & -\Im h_{i j} \\
\Im h_{i j} & \Re h_{i j}
\end{array}\right)
$$

where $\Re h_{i j}$ and $\Im h_{i j}$ denote the real and the imaginary part of $h_{i j}$, respectively. Obviously, the matrix dimensions are extended to $n_{T} \leftarrow 2 n_{T}$ and $n_{R} \leftarrow 2 n_{R}$. Number of bits per real dimension is $M_{c} \leftarrow M_{c} / 2$. The complex vector of the symbols is extended to the real vector $\mathbf{s} \leftarrow\left(\mathbf{s}_{i}\right)$ as follows

$$
\mathbf{s}_{i}=\left(\begin{array}{c}
\Re s_{i} \\
\Im s_{i}
\end{array}\right) .
$$

The other complex vectors $\mathbf{n}$ and $\mathbf{y}$ of the model (1) are extended in the same way. The derivations of the paper for the complex model also hold for the extended real model.

\section{B. $\Lambda$ as an Additive Metric}

In order to compute $\Lambda$ sequentially over the branches of the tree, we use the $\mathrm{QR}$ decomposition of the matrix $\mathbf{H}=\mathbf{Q R}$, where $\mathbf{R}=\left(r_{i j}\right)$ is an $n_{T} \times n_{T}$ triangular matrix and $\mathbf{Q}$ is an $n_{R} \times n_{T}$ orthogonal matrix. The distance $\|\mathbf{y}-\mathbf{H s}\|^{2}$ can 
be written as $\|\mathbf{z}-\mathbf{R} \mathbf{s}\|^{2}$ in which $\mathbf{z}=\mathbf{Q}^{\mathrm{H}} \mathbf{y}$. By expanding $\Lambda$ in (4) to the summation form, we have an additive metric

$$
\Lambda\left(\mathbf{x}, \boldsymbol{\lambda}_{A} ; \mathbf{z}, \mathbf{R}\right)=\sum_{i=1}^{n_{T}} \Lambda_{i}
$$

with the metric increments

$$
\Lambda_{i}=-\frac{1}{2 \sigma^{2}}\left(z_{i}-\sum_{j=i}^{n_{T}} r_{i j} s_{j}\right)^{2}+\frac{1}{2} \sum_{j=1}^{M_{c}} x_{i j} \lambda_{A}\left(x_{i j}\right)
$$

in which $x_{i j}=x_{(i-1) M_{c}+j}$ for $i=1,2, \ldots, n_{T}$ and $j=1,2, \ldots, M_{c}$. Referring to (10), $\Lambda_{i}$ only depends on the transmit symbols $s_{j}$ for $j \geq i$ and the corresponding a priori values.

\section{The Proposed Algorithm}

The proposed algorithm searches for the best candidate points inside a sphere of radius $C$. This means $\|\mathbf{z}-\mathbf{R s}\|^{2}<$ $C^{2}$ or

$$
\sum_{i=1}^{n_{T}}\left(z_{i}-\sum_{j=i}^{n_{T}} r_{i j} s_{j}\right)^{2} \leq C^{2} .
$$

Obviously, each element of the summation should satisfy the inequality. Starting from the $n_{T}$ th element and assuming $r_{n_{T} n_{T}}>0^{1}$, we conclude that $s_{n_{T}}$ belongs to the following interval [6]

$$
\left\lceil\frac{-C+z_{n_{T}}}{r_{n_{T} n_{T}}}\right\rceil \leq s_{n_{T}} \leq\left\lfloor\frac{C+z_{n_{T}}}{r_{n_{T} n_{T}}}\right\rfloor .
$$

where $\lceil\cdot\rceil$ and $\lfloor\cdot\rfloor$ denote the ceiling and floor operations. For every $s_{n_{T}}$ satisfying (12), we define $C_{n_{T}-1 \mid n_{T}}^{2}=C^{2}-\left(z_{n_{T}}-\right.$ $\left.r_{n_{T} n_{T}} s_{n_{T}}\right)^{2}$ and $z_{n_{T}-1 \mid n_{T}}=z_{n_{T}-1}-r_{n_{T}-1, n_{T}} s_{n_{T}}$. Noting the $\left(n_{T}-1\right)$ th term in (11), we conclude that $s_{n_{T}-1}$ belongs to the following interval

$$
\begin{aligned}
\left\lceil\frac{-C_{n_{T}-1 \mid n_{T}}+z_{n_{T}-1 \mid n_{T}}}{r_{n_{T}-1, n_{T}-1}}\right\rceil & \leq s_{n_{T}-1} \\
& \leq\left\lfloor\frac{C_{n_{T}-1 \mid n_{T}}+z_{n_{T}-1 \mid n_{T}}}{r_{n_{T}-1, n_{T}-1}}\right\rfloor .
\end{aligned}
$$

One can continue in a similar manner for $s_{n_{T}-2}$, and so on until $s_{1}$, thereby going through all the points inside the sphere of radius $C$.

The radius $C$ is chosen based on the statistics of the noise [30]. Note that $\frac{1}{2 \sigma^{2}}\|\mathbf{n}\|^{2}=\frac{1}{2 \sigma^{2}}\|\mathbf{y}-\mathbf{H x}\|^{2}$ is a $\mathcal{X}^{2}$ random variable with $n_{R} / 2$ degree of freedom. Thus, we may choose the radius proportional to the variance of the noise [16], [30],

$$
C^{2}=K_{r} n_{R} \sigma^{2}
$$

where the factor $K_{r}$ controls the tradeoff between complexity and performance.

Noting that the number of constellation points bounded within a sphere of a given radius is inversely proportional to

\footnotetext{
${ }^{1}$ Since $\mathbf{R}$ is real, without loss of generality, we assume $r_{i i}>0$ for $i=$ $1, \ldots, n_{T}$
}

$\operatorname{det}\left(\mathbf{H H}^{\mathrm{H}}\right)$, we select $m=\min \left(K_{m} / \operatorname{det}\left(\mathbf{H} \mathbf{H}^{\mathrm{H}}\right), m_{\max }\right)$. The values of $K_{r}, K_{m}$ and $m_{\max }$ are experimentally selected to minimize the complexity, while achieving the best possible performance. Note that imposing an upper bound on the allowed $m$ limits the peak complexity. Noting above steps, the proposed algorithm can be summarized as follows:

Input. Inputs are $\mathbf{H}, \mathbf{y}, \boldsymbol{\lambda}_{A}, \sigma^{2}, n_{T}, n_{R}$, and $M_{c}$, all in the real space. Maximum number of nodes at each level of the tree is $m$. The $2^{M_{c}}$ points of the real constellation are the elements of the set $\mathcal{Q}_{r}$.

Step 1. (Initialization) Decompose $\mathbf{H}$ to $\mathbf{Q R}$. $\mathbf{z}=\mathbf{Q}^{\mathrm{H}} \mathbf{y}$. Each node has its own level, $i$, metric, $\alpha_{i}$, label $^{2}, \mathbf{s}_{i}=\left(s_{i}, \ldots, s_{n_{T}}\right)$ or $\mathbf{x}_{i}=\left\{\left\{x_{k j}\right\}_{j=1}^{M_{c}} \mid k=\right.$ $\left.i, \ldots, n_{T}\right\}$, sub-sphere radius for the next level, $C_{i-1 \mid i}$, and received signal for the next level, $z_{i-1 \mid i}$. Initialize root node (level $\left.n_{T}+1\right)$ with $\alpha_{n_{T}+1}=0$, $C_{n_{T} \mid n_{T}+1}=C, z_{n_{T} \mid n_{T}+1}=z_{n_{T}}$, and empty label. Set total number of nodes to 1 . Set $i=n_{T}$.

Step 2. (Node extension) For each node of the level $i+1$,

a) (Lower bound) Set $L B=\left[\frac{-C_{i \mid i+1}+z_{i \mid i+1}}{r_{i i}}\right]$.

b) (Upper bound) Set $U B=\left|\frac{C_{i \mid i+1}+z_{i \mid i+1}}{r_{i i}}\right|$.

c) (Extended nodes) Extend the current node to the nodes $s_{i} \in[L B, U B] \cap \mathcal{Q}_{r}$.

d) (Label and metric) For each extended node $s_{i}$ and its corresponding bit label $\left\{x_{i j}\right\}_{j=1}^{M_{c}}$ :

(i) (Labeling ) Label the extended node as $\mathbf{s}_{i}=$ $\left(s_{i}, \mathbf{s}_{i+1}\right)$ or $\mathbf{x}_{i}=\left\{\left\{x_{i j}\right\}_{j=1}^{M_{c}}, \mathbf{x}_{i+1}\right\}$.

(ii) (Metric increment) $\Lambda_{i}=\frac{-1}{2 \sigma^{2}}\left(z_{i \mid i+1}-r_{i i} s_{i}\right)^{2}+$ $\frac{1}{2} \sum_{j=1}^{M_{c}} x_{i j} \lambda_{A}\left(x_{i j}\right)$

(iii) (Metric update ) $\alpha_{i}=\alpha_{i+1}+\Lambda_{i}$

Step 3. (Node selection) Among all the nodes of the level $i$, select at most $m$ nodes with the highest metrics. Update number of nodes to the number of the selected nodes. Eliminate other nodes.

Step 4. (Next level) If $i=1$, go to Step 7, otherwise set $i=i-1$.

Step 5. (Update parameters) For each node of the level $i+$ 1:
a) $z_{i \mid i+1}=z_{i}-\sum_{j=i+1}^{n_{T}} r_{i j} s_{j}$.
b) $C_{i \mid i+1}^{2}=C_{i+1 \mid i+2}^{2}-\left(z_{i+1 \mid i+2}-r_{i+1, i+1} s_{i+1}\right)^{2}$.

Step 6. (Loop) Go to Step 2.

Step 7. (List) For each node of the level 1, save its label $\left(s_{1}, \ldots, s_{n_{T}}\right)$ or $\left(x_{1}, \ldots, x_{M_{c} n_{T}}\right)$ and its corresponding metric $\Lambda=\alpha_{1}$ in the set $\mathcal{L}$.

Output. Outputs are the set $\mathcal{L}$ and the corresponding $\Lambda$ values.

Note that the algorithm does not guarantee to deliver a list of $m$ points, unless the initial radius is set sufficiently high.

\section{Approximate APP Value}

After finding the list $\mathcal{L}$ and its corresponding $\Lambda$-values, the list is applied to (3) to compute the APP values of the

\footnotetext{
${ }^{2}$ Label of a node is defined as the path from the root node to the current node.
} 


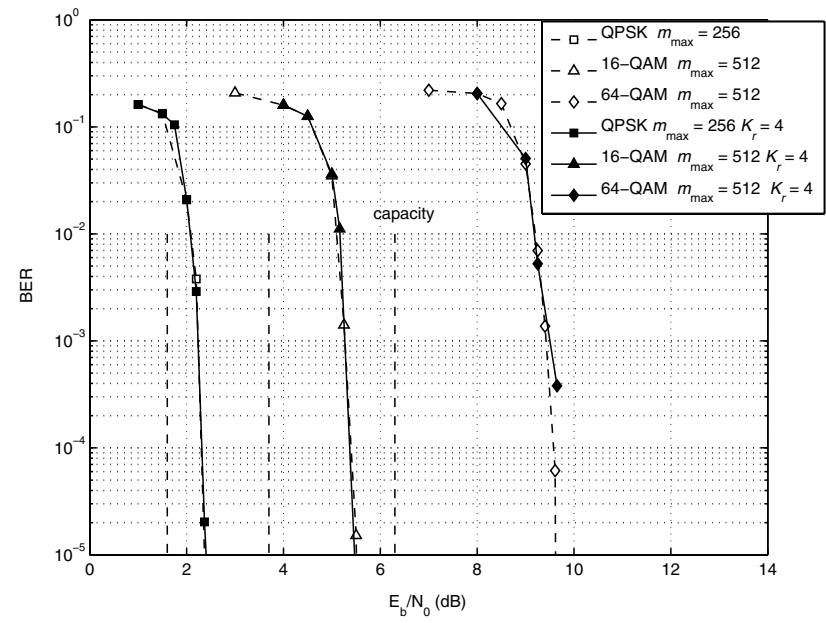

Fig. 2. Performance results for a turbo coded MIMO system with 4 transmit and receive antennas.

transmitted bits. To maintain a reasonable complexity, the exact $\exp (\cdot)$ operation in (3) is implemented in the log-domain by deploying the max- or max ${ }^{*}$-approximation [31]. With the max approximation, the posterior information of $x_{k}$ becomes

$$
\begin{aligned}
\lambda_{D}\left(x_{k} \mid \mathbf{z}\right) \approx & \max _{\mathbf{x} \in \mathbb{X}_{k}^{+} \cap \mathcal{L}}\left\{\Lambda\left(\mathbf{x}, \boldsymbol{\lambda}_{A} ; \mathbf{z}, \mathbf{R}\right)\right\} \\
& -\max _{\mathbf{x} \in \mathbb{X}_{k}^{-} \cap \mathcal{L}}\left\{\Lambda\left(\mathbf{x}, \boldsymbol{\lambda}_{A} ; \mathbf{z}, \mathbf{R}\right)\right\}
\end{aligned}
$$

in which the maximizations are over the points of the list $\mathcal{L}$. If $\mathbb{X}_{k}^{+} \cap \mathcal{L}$ (or $\mathbb{X}_{k}^{-} \cap \mathcal{L}$ ) is empty, then $\lambda_{D}\left(x_{k} \mid \mathbf{z}\right)$ is set to the given minimum (or maximum) value of the LLR [16].

\section{Numerical Results}

Numerical results are provided for the same scenario as described in [16] for a turbo-coded MIMO system. Fig. 2 shows the performance results for a $4 \times 4$ MIMO. There is no degradation for the proposed method in comparison with the $\mathrm{m}$-algorithm and the LSD presented in [16].

The advantage of the proposed method lies in its complexity which is illustrated for a 16-QAM, $4 \times 4$ MIMO system in Fig. 3. As this figure shows, the complexity of the proposed method is much less than the m-algorithm and the LSD. Computational complexity is measured in terms of the number of branches enumerated at each level of the tree. The higher is the number of constellation points and/or the number of antennas, the higher will be the complexity gain achieved due to using the proposed method.

According to Fig. 3, the complexity of the m-algorithm is fixed for all SNR values. The complexity of the m-algorithm is very high for the large SNR values, where the other two methods have lower complexities. Note that the complexity of the m-algorithm is the worst case complexity of the proposed PSD.

As Fig. 3 shows, for low SNR values, the average complexity of the LSD is higher than the worst case complexity of the PSD. Therefore, one advantage of the proposed method is that

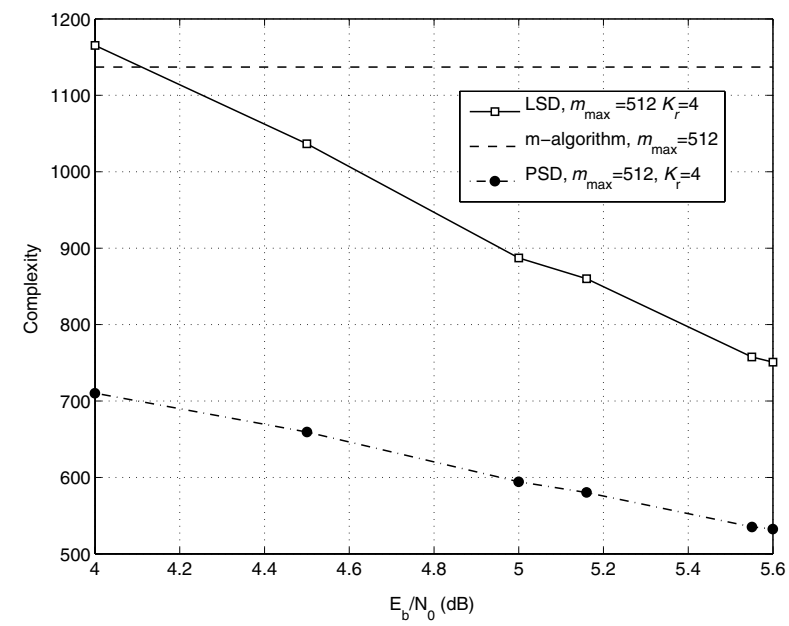

Fig. 3. Complexity of different detection algorithms for a $16-\mathrm{QAM}, 4 \times 4$ MIMO system.

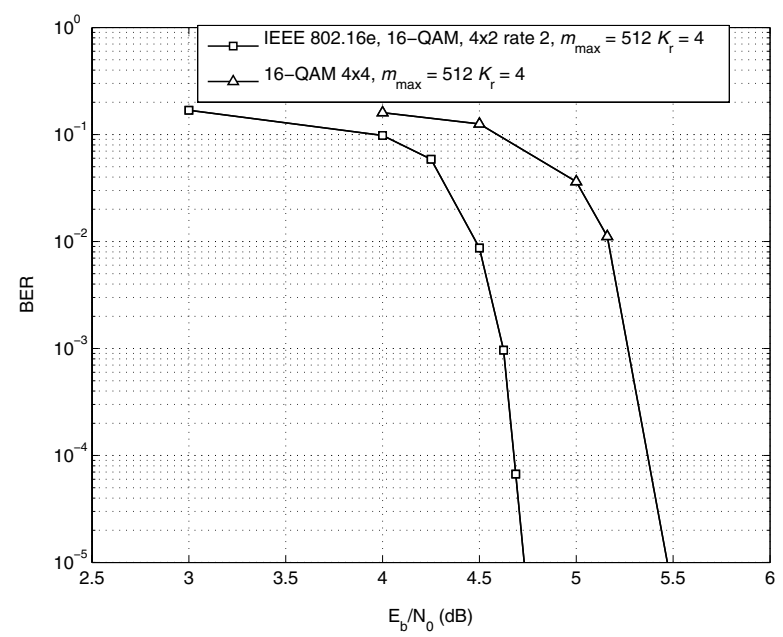

Fig. 4. Performance result for IEEE 802.16e standard with a space-time code of rate 2,16-QAM, $4 \times 2$ MIMO. The result is compared with a 16-QAM $4 \times 4$ MIMO system.

its complexity does not exceed a threshold even at low SNR values where the sphere radius is large.

Fig. 4 is the performance result for a $4 \times 2$ space time code of rate 2 adopted in the IEEE 802.16e standard. The performance result is compared with a 16-QAM, $4 \times 4$ MIMO system (rate 4). The complexity results are similar to those reported in Fig. 3.

\section{CONCLUSION}

A soft MIMO detector is presented which achieves significant complexity gains for a similar performance as the other relevant methods reported in the literature. In addition, the parallel structure of the proposed method is suitable for hardware parallelization. 


\section{REFERENCES}

[1] G. J. Foschini and M. J. Gans, "On the limits of wireless communications in a fading when using multiple antennas," Wireless Pres. Commun., vol. 6, pp. 311-335, 1998.

[2] C. N. Chuah, D. N. C. Tse, J. M. Kahn, and R. A. Valenzuela, "Capacity scaling in MIMO wireless systems under correlated fading," IEEE Trans. Inform. Theory, vol. 48, pp. 637-650, Mar. 2002.

[3] G. J. Foschini, "Layered space-time architecture for wireless communication in a fading environment when using multi-element antennas," Bell Labs. Tech. J., vol. 1, pp. 41-59, 1996.

[4] G. D. Golden, G. J. Foschini, R. A. Valenzuela, and P. W. Wolnianky, "Detection algorithm and initial laboratory results using V-BALST space-time communication architecture," Electron. Lett., vol. 35, pp. 14 16, Jan. 1999

[5] E. Agrell, A. Vardy, and K. Zeger, "Closest point search in lattices," IEEE Trans. Inform. Theory, vol. 48, pp. 2201-2214, Aug. 2002.

[6] M. O. Damen, H. El-Gamal, and G. Cairo, "On maximum-likelihood detection and the search for the closest lattice point," IEEE Trans. Inform. Theory, vol. 49, no. 10, pp. 2389-2402, Oct. 2003.

[7] J. Jalden and B. Ottersten, "On the complexity of sphere decoding in digital communications," IEEE Trans. Signal Processing, vol. 53, pp. 1474-1484, Sept. 2005.

[8] S. Benedetto, G. Montorsi, D. Divsalar, and F. Pollara, "A soft-input soft-output maximum a posterior (MAP) module to decode parallel and serial concatenated codes," NASA JPL TDA Progress Report, pp. 42 127, Nov. 1996.

[9] J. Hagenauer, "The turbo principle: Tutorial introduction and state of the art," in Proc. 1st Int. Symposium on Turbo Codes, Sept. 1997, pp. $1-12$.

[10] M. O. Damen, "Joint coding/decoding in a multiple access system, application to mobile communications," Ph.D. dissertation, Ecole Nationale Superieure des Telecommunications, Oct. 1999.

[11] A. Stefanov and T. Duman, "Turbo-coded modulation for systems with transmit and receive antenna diversity over block fading channels: System model, decoding approaches, and practical considerations," IEEE J. Select. Areas Commun., vol. 19, pp. 958-968, May 2001.

[12] M. Sellathurai and S. Haykin, "Turbo-BLAST for wireless communications: Theory and experiments," IEEE Trans. Signal Processing, vol. 50, pp. 2538-2546, Oct. 2002.

[13] J. Boutros and G. Caire, "Iterative multiuser joint decoding: unified framework and asymptotic analysis," IEEE Trans. Inform. Theory, vol. 48, pp. 1772-1793, July 2002.

[14] C. Berrou, A. Glavieux, and P. Thitimajshima, "Near Shannon limit error-correcting coding and decoding: turbo-codes," in Proc. of IEEE Int. Conf. on Commun., ICC '93, Geneva, May 1993, pp. 1064-1070.

[15] R. G. Gallager, Low-Density Parity-Check Codes. Cambridge, MA: MIT Press, 1963.

[16] B. M. Hochwald and S. ten Brink, "Achieving near-capacity on a multiple-antenna channel," IEEE Trans. Commun., vol. 51, pp. 389-399, Mar. 2003.

[17] J. Boutros, N. Gresset, L. Brunel, and M. Fossorier, "Soft-input softoutput lattice sphere decoder for linear channels," in Proc. of IEEE Global Telecommun. Conf., GLOBECOM '03, vol. 3, Dec. 1-5, 2003, pp. 1583-1587.

[18] S. Baro, J. Hagenauer, and M. Witzke, "Iterative detection of MIMO transmission using a list-sequential (LISS) detector," in Proc. of IEEE Int. Conf. on Commun., ICC '03, Anchorage, AK, May 2003, pp. 2653 2657.

[19] B. Dong, X. Wang, and A. Doucet, "A new class of soft MIMO demodulation algorithms," IEEE Trans. Signal Processing, vol. 51, pp. 2752-2763, Nov. 2003.

[20] Y. L. C. de Jong and T. J. Willink, "Iterative tree search detection for MIMO wireless systems," in Proc. of IEEE 56th Veh. Technol. Conf., VTC '02-Fall, vol. 2, Sept. 2002, pp. 1041-1045 vol.2.

[21] A. B. Reid, A. J. Grant, and A. P. Kind, "Iterative tree search detection for MIMO wireless systems," in Proc. of 4th Australian Commun. Theory Workshop, AusCTW '03, Melbourne, Australia, Feb. 5-7, 2003, pp. 66-69.

[22] J. Anderson and S. Mohan, "Sequential coding algorithms: A survey and cost analysis," IEEE Trans. Commun., vol. 32, pp. 169-176, Feb. 1984.

[23] C. Schlegel, Trellis Coding. New York: IEEE Press, 1997.
[24] R. Wang and G. B. Giannakis, "Approaching MIMO channel capacity with reduced-complexity soft sphere decoding," in Proc. of IEEE Wireless Commun. and Networking Conf., WCNC '04, vol. 3, Mar. 21-25, 2004, pp. 1620-1625.

[25] B. Steingrimsson, T. Luo, and K. M. Wong, "Soft quasi-maximumlikelihood detection for multiple-antenna wireless channels," IEEE Trans. Signal Processing, vol. 51, pp. 2710- 2719, Nov. 2003.

[26] A. Mobasher, M. Taherzadeh, R. Sotirov, and A. K. Khandani, "A near maximum likelihood decoding algorithm for mimo systems based on graph partitioning," in Proc. of IEEE Int. Symp. on Info. Theory, ISIT 05, Adelaide, Australia, Sept. 4-9, 2005.

[27] J. J. Boutros, F. Boixadera, and C. Lamy, "Bit-interleaved coded modulations for multiple-input multiple-output channels," in Proc. of IEEE 6th Int. Symp. on Spread Spectrum Techniques and Applications, vol. 1, NJ, Sept. 6-8, 2000, pp. 123-126 vol.1.

[28] A. Kind and A. Grant, "Efficient list decoding for lattices," in Proc. of 4th Australian Commun. Theory Workshop, AusCTW '03, Melbourne, Australia, Feb. 5-7, 2003, pp. 1-5.

[29] M. Ajtai, "The shortest vector problem in $L_{2}$ is NP-hard for randomized reductions," in Proc. of 30th Annu. ACM Symp. Theory of Comput. (STOC), 1998, pp. 10-19.

[30] B. Hassibi and H. Vikalo, "On the expected complexity of sphere decoding," in Proc. of the 35th Asilomar Conf. on Signals, Systems and Computers, vol. 2, Pacific Grove, CA, Nov. 4-7, 2001, pp. 1051-1055 vol.2.

[31] P. Robertson, E. Villebrun, and P. Hoeher, "A comparison of optimal and sub-optimal MAP decoding algorithms operating in the log domain," in Proc. of IEEE Int. Conf. on Commun., ICC '95, vol. 2, Seattle, June 18-22, 1995, pp. 1009-1013 vol.2. 